Vittalle - Revista de Ciências da Saúde v. 32, n. 2 (2020) 109-120
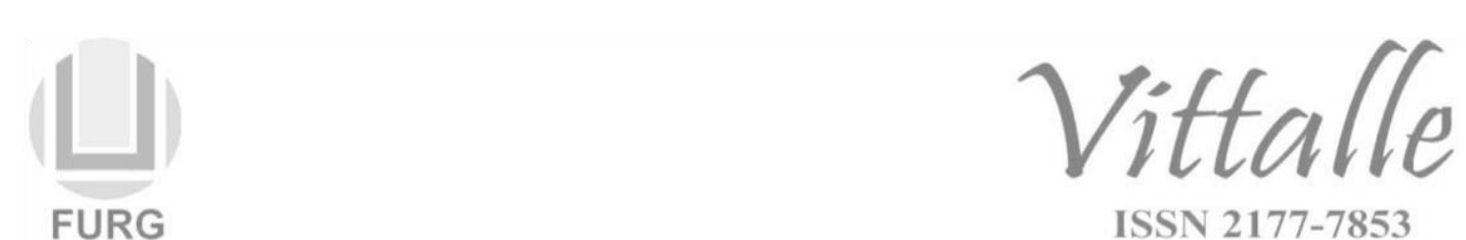

\title{
Aspectos qualitativos do estudo das interações medicamentosas em Hospitais Universitários brasileiros: revisão sistemática
}

\author{
Augusto Santana Palma Silva ${ }^{a^{*}}$, Sheila Torres Feitosa Silva ${ }^{b}$ \\ aPós-graduação em Dinâmicas de Desenvolvimento do Semiárido. Universidade Federal do Vale do São Francisco \\ (UNIVASF), Petrolina, PE, Brasil

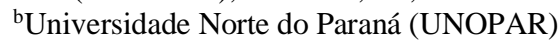

Histórico do Artigo

Recebido em:

$13 / 11 / 2019$

Aceito em:

$29 / 02 / 2020$

\section{Palavras-chave:}

Revisão sistemática;

interações

medicamentosas;

hospital universitário;

equipe

multiprofissional

Keywords:

Systematic review;

drug interactions;

universitary hospital;

multiprofessional

team

\begin{abstract}
RESUMO
Hospitais Universitários são ambientes vinculados a Instituições de Ensino Superior onde são desempenhadas atividades de ensino, pesquisa e assistência. Em função do seu perfil misto para pacientes, que demandam esquemas farmacoterapêuticos diversos, configuram ambiente propício à ocorrência de Interações Medicamentosas, contexto que demanda um processo de cuidado em saúde pautado pela lógica multiprofissional. As atuais revisões sistemáticas da literatura acerca de interações medicamentosas e ambientes hospitalares não abordam aspectos de natureza qualitativa relacionados ao perfil de profissionais envolvidos e a relevância para outras áreas de atuação, objetivo do presente estudo. Conduziu-se uma revisão sistemática baseada na estratégia SPIDER de busca em quatro bancos de dados mediante emprego de nove descritores e operadores booleanos que deveriam compor a seção título de manuscritos disponíveis integralmente em português ou inglês e realizados em hospitais universitários brasileiros. Dezessete citações compõem a amostra final, sendo nove artigos, três dissertações, duas teses e três trabalhos de conclusão de curso. Houve prevalência para estudos realizados por profissionais farmacêuticos, seguido de enfermeiros e médicos. Parcela expressiva dos estudos apresentou baixa qualidade e relevância para outros profissionais, sendo realizados majoritariamente por apenas um tipo de profissional. Foram identificados poucos estudos publicados envolvendo a temática, considerando-se o quantitativo de Hospitais Universitários brasileiros existentes e o período temporal considerado. Estratégias evidenciadas e relacionadas ao enfrentamento da problemática residem em aspectos relacionados ao paciente, ao estabelecimento e aos profissionais, de modo a assegurar a detecção, redução ou eliminação de esquemas terapêuticos potencialmente interativos por parte dos profissionais que os prescrevem, dispensam, preparam e administram.
\end{abstract}

Qualitative aspects of the study of medication interactions in brazilian University Hospitals: a systematic review

\section{ABSTRACT}

University Hospitals are environments linked to Higher Education Institutions where teaching, research and assistance activities are performed. Due to its mixed profile for patients, who require different pharmacotherapeutic regimens, they provide an environment conducive to the occurrence of Drug Interactions, a context that demands a health care process based on multiprofessional logic. Current systematic literature reviews on drug interactions and hospital environments do not address aspects of a qualitative nature related to the profile of professionals involved and the relevance to other areas of activity, objective of the present study. We conducted a systematic review based on the SPIDER strategy of searching four databases by employing nine Boolean descriptors and operators that should compose the title section of manuscripts available entirely in Portuguese or English and performed in Brazilian university hospitals. Seventeen citations make up the final sample, being nine articles, three dissertations, two theses and three final papers. There was prevalence for studies conducted by pharmaceutical professionals, followed by nurses and doctors. A significant portion of the studies presented low quality and relevance to other professionals, being performed mostly by only one type of professional. Few published studies involving the theme were identified, considering the number of existing Brazilian University Hospitals and the time period considered. Strategies evidenced and related to coping with the problem reside in aspects related to the patient, the establishment and the professionals, in order to ensure the detection, reduction or elimination of potentially interactive therapeutic regimens by the professionals who prescribe, dismiss, prepare and administer them.

\footnotetext{
* Autor correspondente: augustolestat@ gmail.com (Silva A.S.P.)
} 


\section{Introdução}

Hospitais Universitários compreendem ambiente onde são desenvolvidas ações estruturadas sob a forma de um tripé Ensino-Pesquisa-Assistência (1). Seu perfil heterogêneo, na forma de distintos serviços (emergencial, cirúrgico, internação, exames), os torna ambientes favoráveis, em função de distintos esquemas farmacoterapêuticos envolvidos, à ocorrência de Interações Medicamentosas alterações no efeito de um medicamento quando (geralmente) na presença de outro (2). Por configurarem-se como Estabelecimentos de Saúde de prestação de serviços de diferentes complexidades, demandam a participação dos mais diversos profissionais, sendo os enfermeiros, farmacêuticos e médicos os mais comumente associados a essas práticas de cuidado, que envolvem a prescrição, dispensação, preparo e administração de esquemas terapêuticos específicos às demandas e necessidades dos pacientes assistidos (3). Chandrasekhar et al. (4) sinalizam que a avaliação criteriosa de prescrições gera impactos significativos na redução da ocorrência de Interações Medicamentosas, e Dechanont et al. (5) evidenciam que a ocorrência deste fenômeno, em especial no ambiente hospitalar, encontra-se associada: a) à visitas e admissões em $0,1 \%$ e $1.1 \%$ dos casos respectivamente e; b) ao desconhecimento por parte dos profissionais que prescrevem (médico), dispensam (farmacêutico), preparam e administram (enfermeiro) os medicamentos. Dito de outra forma: o fenômeno das Interações Medicamentosas (e seus impactos) em Hospitais Universitários deve considerar um trabalho realizado de maneira multiprofissional e sua pesquisa deve pautar-se na produção de conhecimentos de ampla abrangência, de modo a contemplar os mais distintos profissionais envolvidos no exercício de cuidado em saúde.

A literatura científica disponível acerca do tema, na forma de levantamentos sistemáticos $(2,4,5)$, se por um lado não abordam aspectos de natureza qualitativa dos manuscritos (em relação à sua importância multiprofissional), por outro desconsideram o perfil de pesquisadores (profissionais e Instituições de Ensino Superior) envolvidos, bem como o comportamento das publicações ao longo dos anos. O presente estudo busca sumarizar os referidos aspectos de modo a estabelecer, no contexto brasileiro, o perfil qualitativo de pesquisadores envolvidos no estudo de Interações Medicamentosas em Hospitais Universitários brasileiros.

\section{Materiais e Métodos}

Realizou-se uma busca sistemática na literatura científica disponível norteada pela estratégia SPIDER (6) visando responder ao questionamento "qual a literatura científica disponível envolvendo o estudo de (potenciais) interações medicamentosas em Hospitais Universitários brasileiros?". Optou-se por esta estratégia em função da não aplicabilidade do modelo tradicional PICOS, bem como o fato de esta estratégia permitir a recuperação de citações cuja abordagem pudesse ter se dado sob o aspecto de estudos de natureza qualitativa e quantitativa. A estratégia de busca utilizou os descritores (i) interações de medicamentos; (ii) interação medicamentosa; (iii) interações medicamentosas; (iv) hospital universitário; (v) hospitais universitários; (vi) hospital de ensino; (vii) hospitais de ensino; (viii) hospital escola e; (ix) hospitais escola, mediante emprego dos operadores booleanos AND e OR, devendo os resultados compor a seção Título dos manuscritos. A busca foi realizada em agosto de 2019 em um único dia, por um dos revisores, utilizando-se computador pessoal e rede de acesso à internet da Universidade Federal do Vale do São Francisco, em quatro bancos de dados: 
a) Biblioteca Virtual em Saúde; b) Google Acadêmico; c) Portal de Periódicos da CAPES e; d) Scientific Eletronic Library Online (SciELO). Após identificação de citações potencialmente relevantes, procedeu-se à exclusão daquelas em duplicata seguida da leitura de Títulos e Resumos e aplicação dos critérios de inclusão e não inclusão. Foram considerados estudos disponíveis integralmente, publicados a qualquer momento, em português ou inglês, realizados em Hospitais Universitários brasileiros, em formato de artigo original ou monografias (trabalhos de conclusão de curso, dissertação ou tese), sendo desconsiderados manuscritos indisponíveis (ou disponíveis parcialmente), fora da temática de interesse ou sob quaisquer outros formatos de apresentação (artigos de revisão, carta editorial, resumos) e ou não realizados em Hospitais Universitários brasileiros. Após determinação da amostra final, procedeu-se à leitura dos manuscritos em sua íntegra, processo realizado por ambos os revisores e registro de informações (em fichas de confecção própria) referentes à Instituição de Ensino Superior envolvida, aos profissionais envolvidos e a qualidade do estudo realizado - esta última com base no instrumento (adaptado) proposto por Russel e Gregory (7) e composto por nove itens que deveriam ser respondidos com sim, não ou não possível determinar (ou não se aplica). Respostas afirmativas valem um ponto e as demais não pontuam (zero). A qualidade do estudo corresponde ao somatório de respostas afirmativas (quanto maior o número de itens positivados, maior a qualidade), estando estas relacionadas: a) à validade dos resultados (are the findings valid?); b) quais foram estes resultados (what are the findings?) e; c) como estes podem ser aplicados à prática de cuidado ao paciente em um contexto multiprofissional (how can I apply the findings to patient care?). Itens relacionados ao tópico "Os resultados são válidos?" compreendem (i) A pergunta da pesquisa foi clara e adequadamente descrita?; (ii) $\mathrm{O}$ delineamento da pesquisa foi apropriado à pergunta?; (iii) $\mathrm{O}$ método de amostragem foi adequado à pergunta e metodologia?; (iv) Os dados foram coletados e registrados de maneira sistemática? e; (v) Os dados foram analisados de maneira apropriada? O item relacionado ao tópico "Quais foram os resultados?" correspondeu à pergunta (vi) A descrição dos resultados foi completa? O tópico "Como eu posso aplicar estes resultados ao cuidado ao paciente?" compreende os itens: (vii) O estudo possui significado e relevância para a minha prática?; (viii) $\mathrm{O}$ estudo me ajuda a compreender o contexto da minha prática? e; (ix) $\mathrm{O}$ estudo aumenta significativamente o meu conhecimento acerca da minha prática? Esta etapa foi realizada por um dos revisores, cuja formação compreende o Bacharelado em Farmácia.

Divergências entre os revisores não ocorrem durante todo o processo de construção do presente estudo, sendo o manuscrito apresentado elaborado conforme a recomendação PRISMA (8).

\section{Resultados e Discussão}

Com base na estratégia de busca utilizada, foram identificadas 39 citações. Após a exclusão de citações em duplicata (14 citações), leitura de títulos e resumos e aplicação dos critérios para inclusão e não inclusão, oito citações foram excluídas por motivo de indisponibilidade (sete citações) e encontrarem-se fora da temática de interesse (uma citação). A amostra final é composta por 17 citações, sendo nove artigos originais (9-17), três Dissertações de Mestrado (18-20), três Trabalhos de Conclusão de Curso (21-23) e duas Teses de Doutorado $(3,24)$. A Figura 1 exibe o diagrama de fluxo adotado na busca sistemática por manuscritos abordando a temática das interações medicamentosas (potenciais) em Hospitais Universitários brasileiros. 
Descritor: interações de medicamentos OR interação medicamentosa OR interações medicamentosas AND hospital universitário OR hospitais universitários OR hospital de ensino OR hospitais de ensino OR hospital escola OR hospitais escola

Seção: Título

Busca: Agosto de 2019

1 - Biblioteca Virtual em Saúde: 4 citações

2- Google Acadêmico: 22 citações

3 - Portal de Periódicos da CAPES: 10 citações

4. - Scientific Eletronic Library Online: 3 citações

Total de citações: 39

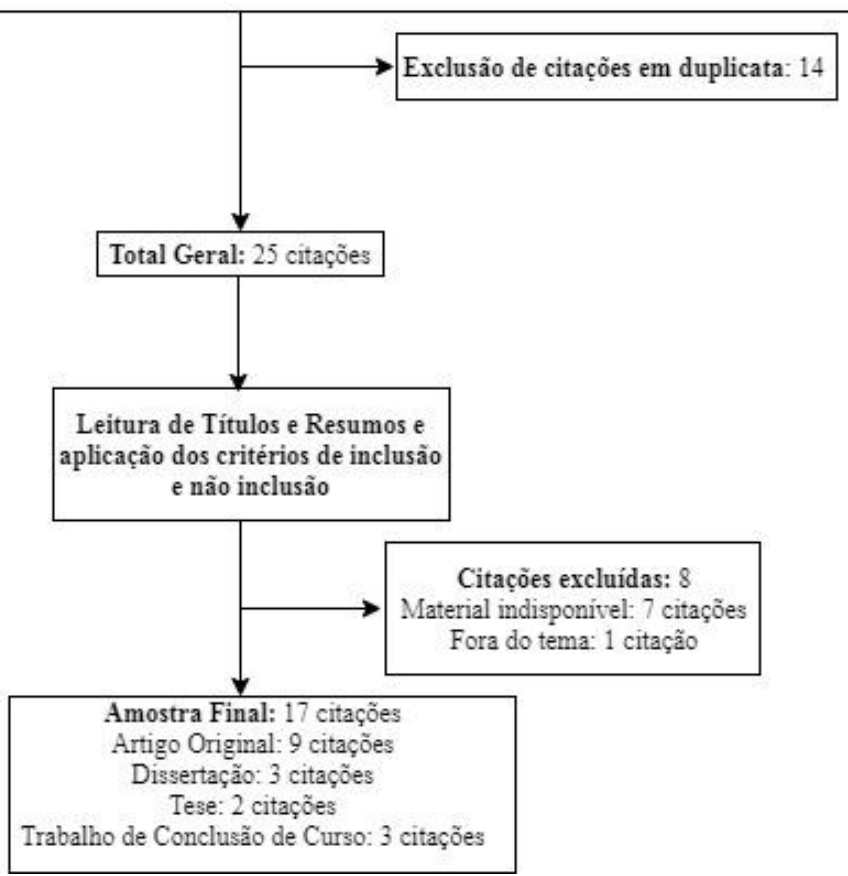

Figura 1 - Diagrama de fluxo da busca sistemática adotada visando identificar manuscritos voltados para o estudo de (potenciais) interações medicamentosas em Hospitais Universitários brasileiros.

O Quadro 1 a seguir sumariza o perfil qualitativo de Instituições de Ensino Superior, profissionais envolvidos e a qualidade dos manuscritos voltados ao estudo do fenômeno de Interações Medicamentosas (potenciais) em Hospitais Universitários brasileiros.

Quadro 1 - Descrição geral dos resultados envolvendo a Instituição de Ensino Superior (IES) vinculada ao Hospital Universitário, perfil profissional envolvido no estudo de (potenciais) interações medicamentosas e a qualidade* dos manuscritos identificados, com base no instrumento adaptado e proposto por Russel e Gregory (7)

\begin{tabular}{|c|c|c|c|c|c|c|c|c|c|c|c|c|}
\hline \multirow{2}{*}{ Referência } & \multirow{2}{*}{ IES } & \multirow{2}{*}{ Profissional } & \multicolumn{10}{|c|}{ Qualidade* } \\
\hline & & & 1 & 2 & 3 & 4 & 5 & 6 & 7 & 8 & 9 & Total \\
\hline Alvim et al. (9) & $\begin{array}{c}\text { Universidade Federal de } \\
\text { Juiz de Fora }\end{array}$ & $\mathrm{ni}$ & 1 & 1 & 1 & 1 & $\mathbf{0}$ & $\mathbf{0}$ & 1 & 1 & 1 & 7 \\
\hline Antunes et al. (10) ${ }^{\mathrm{a}}$ & $\begin{array}{l}\text { Universidade Federal de } \\
\text { São Paulo }\end{array}$ & Enfermagem & 1 & 1 & $\mathbf{0}$ & $\mathbf{0}$ & $\mathbf{0}$ & $\mathbf{0}$ & $\mathbf{0}$ & $\mathbf{0}$ & $\mathbf{0}$ & 2 \\
\hline Backes $(18)^{d}$ & $\begin{array}{c}\text { Universidade Federal de } \\
\text { Santa Catarina }\end{array}$ & Farmácia & 1 & 1 & $\mathbf{0}$ & $\mathbf{0}$ & $\mathbf{0}$ & 1 & 1 & 1 & 1 & 6 \\
\hline Barros $(21)^{\mathrm{m}}$ & Universidade de Brasília & Farmácia & 1 & $\mathbf{0}$ & $\mathbf{0}$ & $\mathbf{0}$ & $\mathbf{0}$ & $\mathbf{0}$ & $\mathbf{0}$ & $\mathbf{0}$ & 1 & 2 \\
\hline $\begin{array}{l}\text { Crussiol-Souza e } \\
\text { Thomson }(11)^{\mathrm{a}}\end{array}$ & $\begin{array}{l}\text { Universidade Estadual de } \\
\text { Londrina }\end{array}$ & $\mathrm{ni}$ & 1 & $\mathbf{0}$ & $\mathbf{0}$ & $\mathbf{0}$ & $\mathbf{0}$ & 1 & 1 & 1 & 1 & 5 \\
\hline Junior et al. (12) ${ }^{\mathrm{a}}$ & $\begin{array}{l}\text { Universidade Federal de } \\
\text { Ciências da Saúde de Porto } \\
\text { Alegre; Universidade } \\
\text { Federal do Rio Grande do } \\
\text { Sul }\end{array}$ & $\begin{array}{l}\text { Medicina; } \\
\text { Estatística }\end{array}$ & 1 & $\mathbf{0}$ & $\mathbf{0}$ & $\mathbf{0}$ & $\mathbf{0}$ & $\mathbf{0}$ & 1 & $\mathbf{0}$ & $\mathbf{0}$ & 2 \\
\hline
\end{tabular}


Continuação

\begin{tabular}{|c|c|c|c|c|c|c|c|c|c|c|c|c|}
\hline \multirow{2}{*}{ Referência } & \multirow{2}{*}{ IES } & \multirow{2}{*}{ Profissional } & \multicolumn{10}{|c|}{ Qualidade* } \\
\hline & & & 1 & 2 & 3 & 4 & 5 & 6 & 7 & 8 & 9 & Total \\
\hline $\operatorname{Lima}(19)^{\mathrm{d}}$ & Universidade de São Paulo & Enfermagem & 1 & $\mathbf{0}$ & $\mathbf{0}$ & 1 & $\mathbf{0}$ & $\mathbf{0}$ & 1 & 1 & 1 & 5 \\
\hline Lima e Cassiani $(13)^{\mathrm{a}}$ & Universidade de São Paulo & Enfermagem & 1 & 1 & 1 & 1 & 1 & 1 & 1 & 1 & 1 & 9 \\
\hline Lopes $(22)^{\mathrm{m}}$ & $\begin{array}{c}\text { Universidade de Santa Cruz } \\
\text { do Sul }\end{array}$ & Farmácia & 1 & $\mathbf{0}$ & 1 & $\mathbf{0}$ & $\mathbf{0}$ & $\mathbf{0}$ & 1 & 1 & 1 & 5 \\
\hline Melo $(24)^{t}$ & Universidade de São Paulo & Farmácia & 1 & $\mathbf{0}$ & $\mathbf{0}$ & $\mathbf{0}$ & $\mathbf{0}$ & $\mathbf{0}$ & 1 & 1 & 1 & 4 \\
\hline Novato $(20)^{\mathrm{d}}$ & Universidade de São Paulo & Farmácia & 1 & $\mathbf{0}$ & $\mathbf{0}$ & $\mathbf{0}$ & $\mathbf{0}$ & $\mathbf{0}$ & $\mathbf{0}$ & $\mathbf{0}$ & $\mathbf{0}$ & 1 \\
\hline Passos et al. (14) ${ }^{\mathrm{a}}$ & $\begin{array}{l}\text { Universidade Federal do } \\
\text { Rio de Janeiro }\end{array}$ & Farmácia & 1 & 1 & 1 & 1 & 1 & 1 & 1 & 1 & 1 & 9 \\
\hline Paula $(3)^{t}$ & $\begin{array}{c}\text { Universidade Federal de } \\
\text { Pernambuco }\end{array}$ & Farmácia & 1 & $\mathbf{0}$ & 1 & $\mathbf{0}$ & $\mathbf{0}$ & $\mathbf{0}$ & 1 & 1 & 1 & 5 \\
\hline Paula et al. $(15)^{\mathrm{a}}$ & $\begin{array}{l}\text { Universidade Federal de } \\
\text { Pernambuco; Universidade } \\
\text { Federal do Vale do São } \\
\text { Francisco }\end{array}$ & ni & 1 & 1 & $\mathbf{0}$ & 1 & $\mathbf{0}$ & $\mathbf{0}$ & 1 & 1 & 1 & 6 \\
\hline Pereira $(23)^{\mathrm{m}}$ & Universidade de Brasília & Farmácia & 1 & $\mathbf{0}$ & $\mathbf{0}$ & $\mathbf{0}$ & $\mathbf{0}$ & $\mathbf{0}$ & $\mathbf{0}$ & $\mathbf{0}$ & $\mathbf{0}$ & 1 \\
\hline $\begin{array}{l}\text { Rodrigues e Pinho } \\
(16)^{\mathrm{a}}\end{array}$ & Universidade de Brasília & $\begin{array}{l}\text { Enfermagem; } \\
\text { Farmácia }\end{array}$ & 1 & 1 & $\mathbf{0}$ & $\mathbf{0}$ & $\mathbf{0}$ & $\mathbf{0}$ & 1 & 1 & 1 & 5 \\
\hline Sousa et al. (17) ${ }^{\mathrm{a}}$ & $\begin{array}{l}\text { Centro Universitário INTA; } \\
\text { Universidade de Fortaleza }\end{array}$ & $\begin{array}{l}\text { Farmácia; } \\
\text { Medicina }\end{array}$ & 1 & $\mathbf{0}$ & $\mathbf{0}$ & $\mathbf{0}$ & $\mathbf{0}$ & $\mathbf{0}$ & 0 & 0 & 1 & 2 \\
\hline
\end{tabular}

Legenda: ${ }^{\text {a: }}$ artigo; ${ }^{\text {}}$ : dissertação; ${ }^{\mathrm{m}}$ : monografia; ${ }^{\text {: }}$ tese; ni: não informado; *foram considerados nove itens, que deveriam ser respondidos com "sim", "não" ou "não possível determinar". Respostas "sim" equivalem a um ponto, ao passo que as demais não pontuam (valor igual a zero). Quanto maior o número de respostas "sim", maior a qualidade do estudo. Os itens em questão encontram-se discriminados na seção Materiais e Métodos.

Alvim et al. (9) avaliaram, em estudo transversal retrospectivo, a ocorrência de Interações Medicamentosas Potenciais em prescrições de pacientes em Unidade de Terapia Intensiva (UTI) do Hospital Universitário da Universidade Federal de Juiz de Fora (Minas Gerais) com ênfase em antimicrobianos. Não foi informado o perfil dos profissionais envolvidos. Análise incompleta e divulgação parcial de informações atribuíram ao estudo uma qualidade igual a sete, dentre os nove itens avaliados.

Antunes et al. (10) estudaram o fenômeno das Interações Medicamentosas Potenciais em prontuários médicos de pacientes idosos (idade igual ou superior a 60 anos) internados no setor de Emergências Clínicas do Hospital São Paulo. Participaram do estudo profissionais da área da Enfermagem. Excetuando-se a formulação da pergunta da pesquisa e delineamento experimental apropriados, os demais itens foram considerados inadequados (dois itens em nove).

Backes (18), em sua Dissertação de Mestrado em Farmácia, avaliou a ocorrência de Interações Medicamentosas Potenciais em prescrições médicas de pacientes atendidos no Hospital Universitário da Universidade Federal de Santa Catarina. Amostragem, coleta e análise dos dados foram descritos inapropriadamente, estando o estudo adequado em seis dos nove itens.

Barros (21), em seu Trabalho de Conclusão de Curso em Farmácia, fruto de pesquisa realizada entre os anos 2013 e 2016 no ambulatório do Hospital Universitário de Brasília, averiguou Interações Medicamentosas Potenciais no contexto de diabéticos tipo 2 em uso de medicamentos anti-hipertensivos, hipoglicemiantes, antihiperglicemiantes e hipolipemiantes. A formulação da pesquisa adequada, e o potencial didático e multiprofissional do manuscrito atribuem a esse documento uma qualidade igual a dois (em nove).

Cruciol-Souza e Thomson (11) avaliaram, em estudo retrospectivo, a ocorrência de Interações Medicamentosas Potenciais em prontuários médicos de pacientes internados em um Hospital Universitário de Londrina. O perfil dos profissionais não foi informado. Delineamento do estudo, amostragem, coleta e análise inadequadas atribuem ao manuscrito uma qualidade igual a cinco (dos nove itens).

Junior et al. (12) avaliaram a ocorrência, em estudo retrospectivo, de Interações Medicamentosas Potenciais em prontuários de pacientes em alas de enfermaria clínica e 
cirúrgica de um Hospital Universitário localizado em Porto Alegre. Participaram do estudo profissionais da área médica e estatística. Pergunta formulada apropriadamente e relevância multiprofissional tornam o estudo adequado em dois dos nove itens considerados.

A Dissertação de Mestrado de Lima (19) corresponde à pesquisa descrita a seguir, na forma de artigo de autoria de Lima e Cassiani (13), não sendo apresentados os resultados gerais do estudo. A avaliação da estrutura do manuscrito, com base na ferramenta proposta por Russel e Gregory (7), evidenciou limitações relacionadas ao delineamento, amostragem, análise e discussão dos resultados. $\mathrm{O}$ manuscrito foi considerado apto em cinco dos nove itens considerados.

Lima e Cassiani (13), profissionais da área da Enfermagem, avaliaram a ocorrência de Interações Medicamentosas Potenciais em estudo transversal de duração de um ano envolvendo análise de prontuários médicos de pacientes em Unidade de Terapia Intensiva do Hospital Universitário do Ceará. O estudo foi considerado adequado em todas as variáveis propostas por Russel e Gregory (7).

Lopes (22), em sua monografia do curso de Farmácia, avaliou o fenômeno das Potenciais Interações Medicamentosas envolvendo, retrospectivamente, análise prescrições de antimicrobianos destinada a pacientes da Unidade de Terapia Intensiva Neopediátrica de um Hospital de Ensino em Santa Cruz do Sul durante janeiro e dezembro de 2016. Delineamento, amostragem, coleta e análise de resultados tornam o estudo adequado em cinco dos nove itens considerados.

Melo (24), em sua Tese de Doutoramento em Farmácia, avaliou retrospectivamente prescrições de pacientes (adultos e idosos) da Clínica Médica do Hospital Universitário da Universidade de São Paulo visando desenvolver um instrumento preditivo de Reações Adversas Evitáveis no contexto farmacoterapêutico. Foi considerado adequado quanto à formulação da pergunta, relevância do estudo, aplicabilidade do conhecimento a outras áreas e potencial didático. Atendeu a quatro dos nove itens considerados.

Novato (20), em sua Dissertação de Mestrado em Farmácia, realizou estudo de natureza observacional e prospectiva no Ambulatório Didático de Geriatria do Hospital das Clínicas (São Paulo) com objetivo de identificar Potenciais Interações Medicamentosas clinicamente significantes. Excetuando-se a formulação da pergunta da pesquisa, os demais itens foram considerados inadequados.

Passos et al. (14) investigaram o fenômeno das Interações Medicamentosas em estudo prospectivo envolvendo pacientes de ambos os sexos internados na Clínica médica do Hospital Universitário Clementino Fraga Filho. Profissionais da área da Farmácia, apresentaram um manuscrito adequado em todos os itens avaliados.

Os resultados fruto da pesquisa de Tese de Doutorado em Farmácia de Paula (3) serão descritos a seguir, sob a forma do manuscrito de autoria de Paula et al. (15). A Tese em questão foi considerada inadequada em quatro (delineamento, coleta, análise e divulgação dos resultados) dos nove itens avaliados.

Paula et al. (15) avaliaram, em estudo transversal prospectivo, a ocorrência de Interações Medicamentosas em prescrições de pacientes em Unidade de Terapia Intensiva (UTI) de um Hospital Universitário de Recife sobre a perspectiva de condições de morbidade para hepatopatias e nefropatias. Perfil profissional não foi informado. Amostragem, análise e divulgação dos resultados foram consideradas inadequadas. $\mathrm{O}$ estudo foi considerado apropriado em seis dos nove itens avaliados.

Pereira (23), em sua monografia em Farmácia desenvolvida durante pesquisa realizada entre agosto de 2013 e abril de 2016 no ambulatório do Hospital Universitário de Brasília, entrevistou pacientes portadores de Diabetes Mellitus a fim de verificar a ocorrência do fenômeno das Interações Medicamentosas envolvendo o uso de fármacos 
hipoglicemiantes e adesão destes pacientes à farmacoterapia. $\mathrm{O}$ estudo apresentou como aspectos adequados a pergunta da pesquisa apenas.

Rodrigues e Pinho (16) avaliaram a ocorrência das Interações Medicamentosas em prontuários de pacientes com idade situada entre 29 dias e 18 anos incompletos em Unidade de Pediatria Clínica de um Hospital Universitário em Brasília, com enfoque na determinação do uso de medicamentos não autorizados e não padronizados para a área pediátrica. Os profissionais da área de Enfermagem e Farmácia apresentaram um manuscrito cuja limitações envolveram inadequações quanto à amostragem, coleta, análise e divulgação dos resultados (atendendo a cinco dos nove itens considerados).

Sousa et al. (17) investigaram, em estudo retrospectivo, a ocorrência de Interações Medicamentosas Potenciais em prontuários de pacientes em Unidade de Terapia Intensiva Adulto em um Hospital Universitário da Universidade do Ceará. O estudo, realizado por profissionais da área Farmacêutica e Médica, apresentou-se como manuscrito adequado quanto à formulação da pergunta e capacidade de ampliar significativamente o conhecimento dos seus leitores (dois em nove dos itens avaliados)

Os resultados permitem verificar, convergência quanto a aspectos positivos e negativos comuns, seja em relação aos artigos e aos Trabalhos de Conclusão de Curso em separado ou em conjunto: a) todos os artigos foram considerados adequados quanto à pergunta de pesquisa (item 1); b) nenhum artigo apresentou aspectos negativos em comum; c) todos os Trabalhos de Conclusão de Curso foram considerados adequados quanto à pergunta de pesquisa (item 1); d) todos os Trabalhos de Conclusão de Curso foram considerados inadequados quanto à análise dos dados realizada (item 5); e) os artigos e Trabalhos de Conclusão de Curso não apresentaram aspecto negativo em comum. Observa-se expressiva participação do profissional farmacêutico $(3,14,16-18$, 20-24), seguido dos de enfermagem $(10,13,16,19)$ e, finalmente, da medicina (17). Os resultados do presente estudo sugerem a pouca comunicação entre os distintos profissionais, haja vista que apenas dois estudos $(16,17)$ foram (aparentemente) conduzidos por profissionais de distintas áreas. Ainda, convém destacar o reduzido número de publicações acerca do tema, considerando o fato de não haver limite para data de publicação e as estratégias de buscas empregadas. A Figura 2 apresenta o comportamento gráfico do número de publicações por ano de manuscritos abordando a temática das Interações Medicamentosas em Hospitais Universitários brasileiros. $\mathrm{O}$ ano de 2015 apresenta o maior número de publicações (iniciando-se em 2004) acerca do tema, com quatro manuscritos $(3,9,10,14)$. 


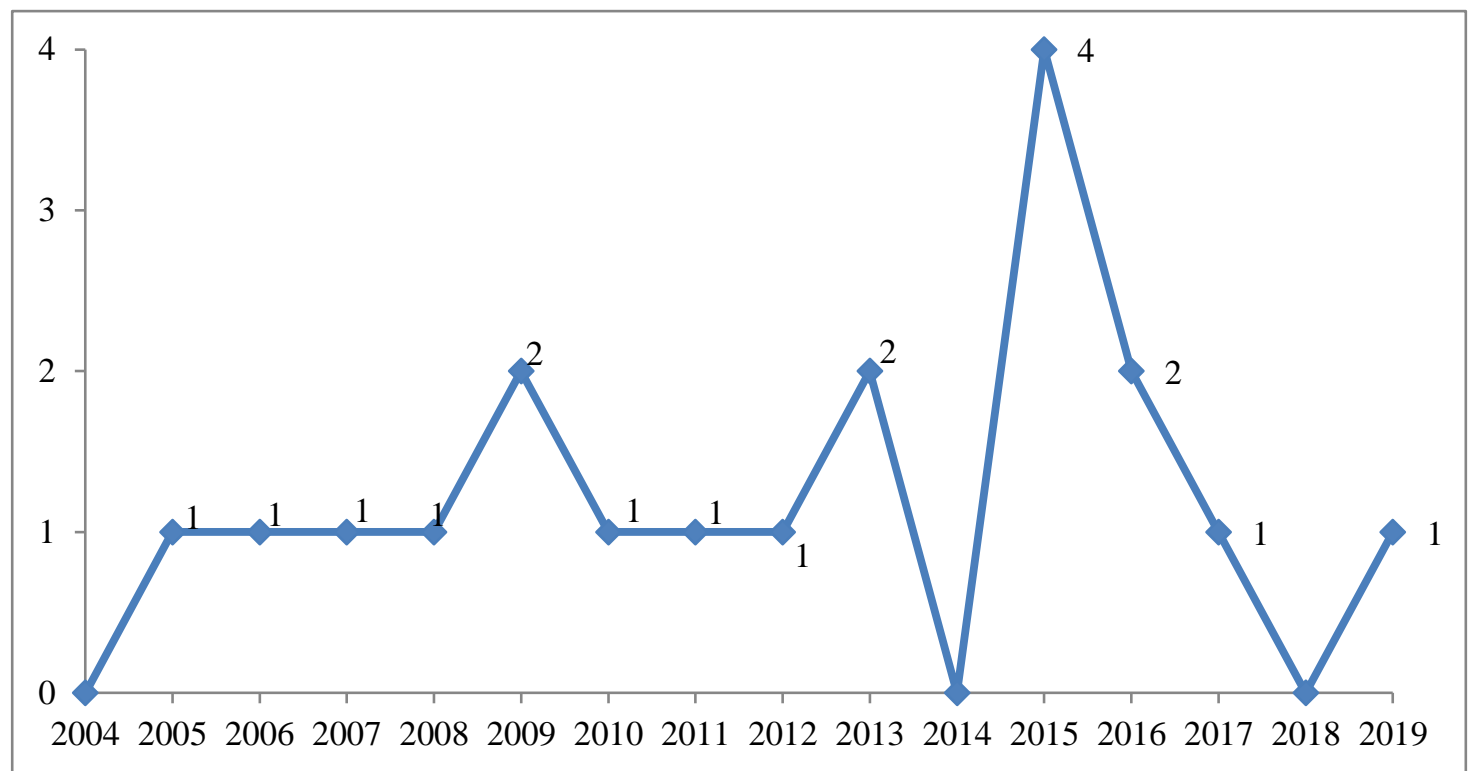

Figura 2 - Número de publicações (Eixo y) por ano (Eixo x) de manuscritos publicados abordando o estudo de Interações Medicamentosas em Hospitais Universitários brasileiros

Aspectos de relevância multiprofissional depreendidos a partir da leitura dos manuscritos residem em variáveis relacionadas: a) ao horário da administração dos medicamentos (relacionada a possibilidade de ocorrência de uma interação medicamentosa), pouco aprofundado ou desconsiderado nos estudos (um dos estudos observou que o horário de administração era sempre o mesmo); b) a ausência (ou reduzida expressividade) de conduta tecnológica, educacional e organizacional nos Hospitais Universitários associado às prescrições (legibilidade, forma farmacêutica, dose, abreviaturas etc.) e; c) à importância de conhecimento acerca dos principais aspectos relacionados às interações medicamentosas (quanto a sua natureza e recomendação) $(3,5)$.

A detecção precoce de Interações Medicamentosas cria cenário favorável à prevenção, redução e ou eliminação de situações cujos desfechos estariam associados ao insucesso farmacoterapêutico e ou surgimento de reações indesejáveis. Evidências relacionadas a fraquezas no contexto da prática clínica incluem: a) a má abordagem do tema durante a graduação; b) a falta de interesse por parte dos profissionais em buscar um maior aprofundamento; c) baixa capacidade nos atuais profissionais em identificar potenciais interações em função dos pares de medicamentos e, finalmente; d) o excesso de informações disponíveis, que dificultam a pesquisa, seleção e síntese de informações direcionadas à tomada de decisão. Sistemas informatizados poderiam minimizar esse problema, apesar do custo envolvendo estes softwares. Em geral, o percentual de estabelecimentos a apresentar a completa implantação destes sistemas é baixo, o que ratifica a importância da condução de pesquisas em caráter multiprofissional, potencializando as competências individuais de cada profissional e minimizando a interferências das ameaças naturais $(3,5,15)$. Dito de outra forma: são necessárias estratégias relacionadas (i) aos pacientes; (ii) aos Hospitais Universitários e; (iii) aos profissionais. 


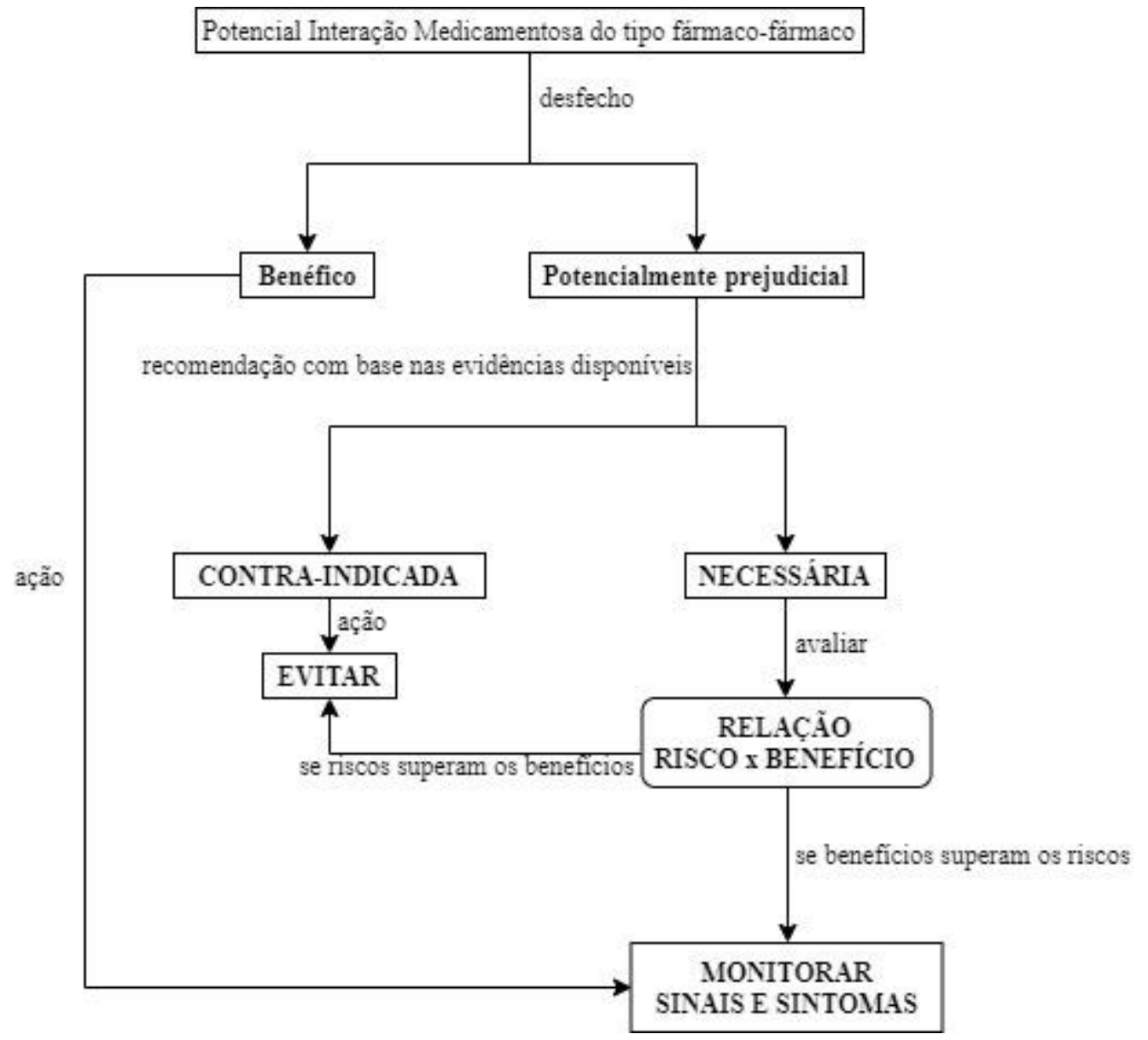

Figura 3 - Relação risco x benefício envolvendo Interações Medicamentosas e seus desfechos para tomada de decisão na prática clínica. Fonte: Adaptado de Paula (3).

Estratégias relacionadas ao paciente podem envolver: a) uso combinado com precaução e, dentro do possível, recomendar a não combinação; b) monitorar sinais sintomas; c) mudar horário de administração e; d) reajuste de dose (menor dose, mas com efeito terapêutico para um ou ambos os fármacos). Fato importante de ser observado é que os aplicativos de detecção analisam pares de medicamentos, mas não a interação entre três ou mais espécies simultaneamente (aparentemente há essa escassez na literatura). Estratégias relacionadas ao Estabelecimento podem envolver: a) prescrição em formato eletrônico (evitando rasuras e ou ausência de informações dose, via de administração, horário); b) adoção de protocolos clínicos; c) padronização de processos e; d) uso de softwares para identificação de Potenciais Interações. Estratégias relacionadas aos Profissionais, finalmente, podem envolver: a) treinamento dos profissionais responsáveis pela prescrição, dispensação, preparação e administração e; b) atuação mais expressiva destes no contexto clínico de cuidado $(3,4)$. 


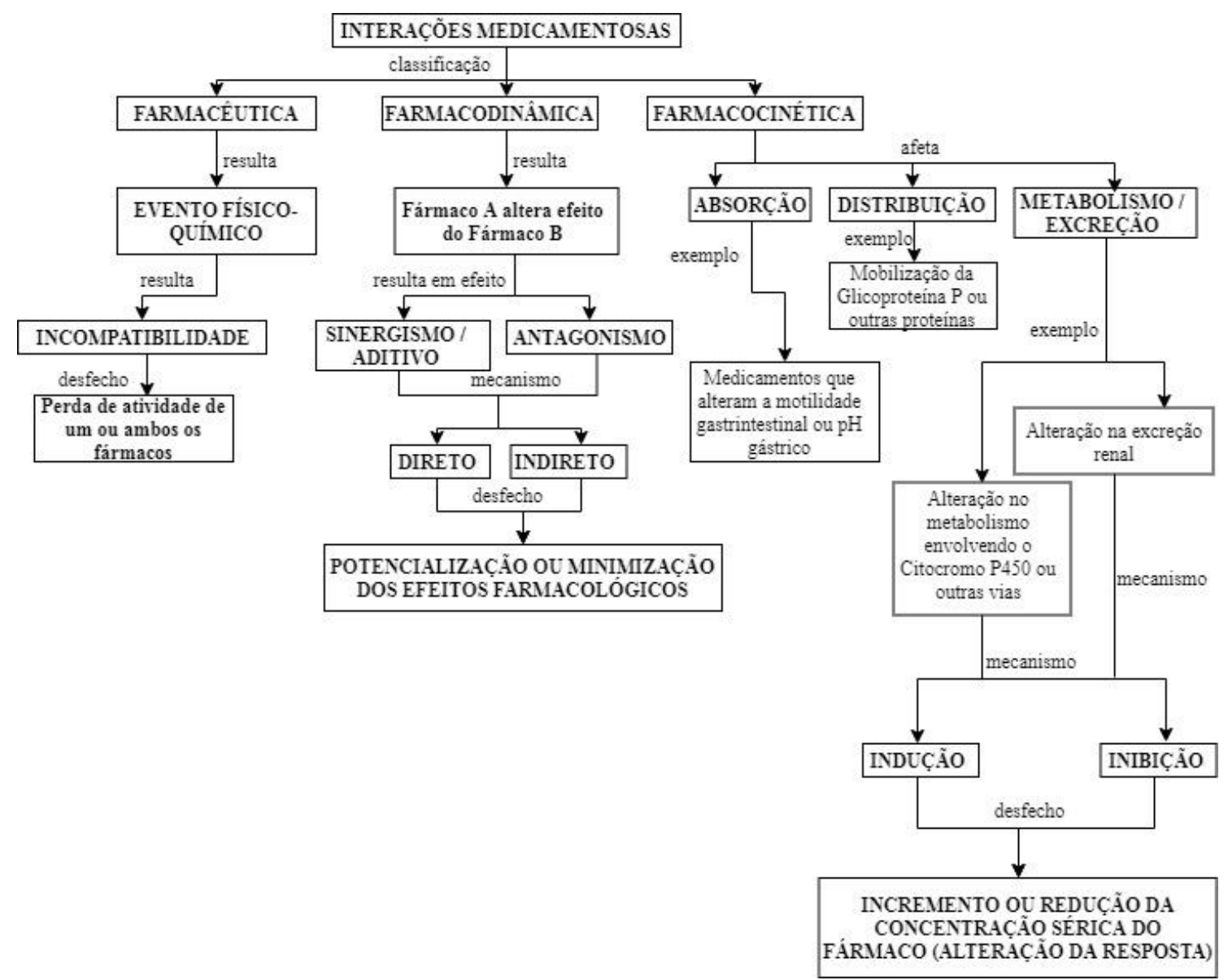

Figura 4 - Classificação das Interações Medicamentosas evidenciando seus desfechos no contexto clínico, evidenciando a possibilidade de intervenção dos profissionais que prescrevem (médicos), dispensam (farmacêuticos), preparam e administram (enfermeiros) medicamentos. Fonte: Adaptado de Paula (3).

O presente estudo, e ainda que tenha considerado maior variação para os descritores empregados (seja para a expressão relacionada às interações medicamentosas, seja para o lócus de estudo), apresenta como limitações o não uso de citações indiretas, o não emprego de outros idiomas, o número de bases consultadas e o local onde os descritores empregados deveriam estar. Futuros estudos, assim, deverão considerar estas limitações durante a elaboração da estratégia de busca sistemática, assegurando maior universo amostral.

\section{Considerações finais}

Hospitais Universitários constituem lócus privilegiado de pesquisa acerca do fenômeno das Interações Medicamentosas, em função do seu heterogêneo perfil de indivíduos assistidos e da equipe de profissionais envolvida neste processo de cuidado (1). Apesar da participação de distintos profissionais neste processo, a literatura científica acerca do estudo das interações em Hospitais Universitários é composta (em sua maioria) por (reduzido número de) estudos não multiprofissionais, apresentados sob a forma de manuscritos de baixa qualidade e relevância para outros profissionais.

O conhecimento geral acerca das potenciais interações e seus mecanismos constitui variável estratégica relacionada à segurança do paciente uma vez que permitirá aos profissionais envolvidos (medicina, farmácia e enfermagem) minimizar ou até mesmo 
eliminar a sua ocorrência, visto ser um fenômeno possível de previsão nos momentos de prescrição, dispensação e administração de medicamentos (3).

Futuros estudos envolvendo a temática das Interações Medicamentosas devem ser conduzidos em Hospitais Universitários brasileiros em respeito e sintonia à logica de pesquisa multiprofissional, uma vez que a pesquisa, uma vez institucionalizada neste ambiente, corrobora para um processo de formação científica não apenas na graduação, mas também em ambientes de pós-graduação (em conformidade às diretrizes estipuladas a esses Estabelecimentos de Saúde). Ainda, esse contato com o ambiente de pesquisa está em sintonia ao processo de formação de profissionais reflexivos, resolutivos e críticos em seus respectivos cenários de práticas, além de poder vir a atuar como catalizador, despertando o interesse pela carreira científica na área - componente estratégico ao desenvolvimento do país $(1,3)$.

\section{Referências}

1. Araújo KM, Leta J. Os hospitais universitários federais e suas missões institucionais no passado e no presente. História, Ciências, Saúde 2014; 21(4): 1261-1281.

2. Amaral RO. Prevalência das interações medicamentosas clinicamente manifestada em pacientes hospitalizados: uma revisão sistemática. São Cristóvão: Universidade Federal de Sergipe, 2016. Trabalho de Conclusão de Curso em Farmácia.

3. Paula VC. Avaliação dos possíveis eventos clínicos adversos decorrentes de interações medicamentosas potenciais em pacientes internados na UTI de um hospital universitário da cidade do Recife. Recife: Universidade Federal de Pernambuco, 2015. Tese em Ciências Farmacêuticas.

4. Chandrasekhar D, Nair AS, Mathew APR, Baby A, Manikandan A. A prospective study on clinically significant drug-drug interactions by Systematic Review of case files in a South Indian Tertiary Care Hospital. Journal of Young Pharmacy 2019;11(3): 285-290.

5. Dechanont S, Maphanta S, Butthum B, Kongkaew C. Hospital admissions/visits associated with drug-drug interactions: a systematic review and meta-anlaysis. Pharmacoepidemiology and Drug Safety 2014;23: 489-497.

6. Cooke A, Smith D, Booth A. Beyonde PICO: the SPIDER tool for qualitative evidence synthesis. Qualitative Health Research 2012;22(10): 1435-1443.

7. Russell CK, Gregory DM. Evaluation of qualitative research studies. Evidence Based Nursing 2003;36(6): 1-5.

8. Galvão TF, Pansani TSA, Harrad D. Principais itens para relatar Revisões sistemáticas e Metaanálises: A recomendação PRISMA. Epidemiologia e Serviços de Saúde 2015;24: 335-342.

9. Alvim MM, Silva LA, Leite ICG, Silvério MS. Eventos adversos por interações medicamentosas potenciais em unidade de terapia intensiva de um hospital de ensino. Revista Brasileira de Terapia Intensiva 2015;27(4): 353-359.

10. Antunes JFS, Okuno MFP, Lopes MCBT, Campanharo CRV, Batista REA. Interação medicamentosa em idosos internados no serviço de emergência de um hospital universitário. Revista Mineira de Enfermagem 2015;19(4): 907-912.

11. Cruciol-Souza JM, Thomson JC. A pharmacoepidemiologic study of drug interactions in a brazilian teaching hospital. CLINICS 2006;61(6): 515-520.

12. Junior FP et al. Potenciais interações medicamentosas em prescrições de um hospital-escola de Porto Alegre. Revista da AMRIGS 2009;53(3): 251-256.

13. Lima REF, Cassiani SHB. Interações medicamentosas potenciais em pacientes de unidade de terapia intensiva de um hospital universitário. Revista Latino-americana de Enfermagem 2009;17(2): 1-7.

14. Passos MMB, Santos RC, Bergamini VG, Souza DC. Interações medicamentosas em pacientes internados na clínica médica de um hospital de ensino e fatores associados. Revista Brasileira de Farmácia 2012;93(2): 450-456.

15. Paula VC, Barreto RR, Santos EJV, Silva AS, Maia MBS. Avaliação de eventos clínicos adversos decorrentes de interações medicamentosas em uma unidade de terapia intensiva de um hospital universitário. Boletim Informativo Geum 2015;6(3): 83-90. 
16. Rodgrigues MCS, Pinho DLM. Polifarmacoterapia e interações medicamentosas: perfil de prescrições pediátricas de um hospital de ensino. Revista Eletrônica Gestão e Saúde 2013;4(1): 13351349.

17. Sousa AB, Oliveira JDML, Cavalcante ALC, Nobre CA, Melo OF, Siqueira RMP. Análise de interações medicamentosas potenciais em pacientes de unidade de terapia intensiva de um hospital de ensino de Sobral. Revista Eletrônica Acervo Saúde 2019;17: 1-9.

18. Backes P. Identificação e avaliação de potenciais interações medicamentosas em prescrições de pacientes internados no hospital universitário da UFSC. Florianópolis: Universidade Federal de Santa Catarina, 2013. Dissertação em Farmácia.

19. Lima REF. Interações medicamentosas potenciais em pacientes de unidade de terapia intensiva de um hospital universitário do Ceará. Ribeirão Preto: Universidade de São Paulo, 2007. Dissertação em Enfermagem Fundamental.

20. Novato ALS. Perfil de prescrição de medicamentos e interações medicamentosas em pacientes acima de 60 anos atendidos em hospital universitário: uma contribuição à farmacovigilância. São Paulo: Universidade de São Paulo, 2005. Dissertação em Farmácia.

21. Barros TS. Análise das interações medicamentosas entre anti-hipertensivos, hipolipemiantes e antihiperglicemiantes em diabéticos acompanhados no hospital universitário de Brasília. Brasília: Universidade de Brasília, 2016. Trabalho de Conclusão de Curso em Farmácia.

22. Lopes BL. Avaliação de prescrições de antimicrobianos e suas interações medicamentosas em pacientes da unidade de terapia intensiva neopediátrica de um hospital de ensino. Santa Cruz do Sul: Universidade de Santa Cruz do Sul, 2017. Trabalho de Conclusão de Curso em Farmácia.

23. Pereira LTG. Interações medicamentosas e adesão ao tratamento em portadores de Diabetes Mellitus Tipo 2 acompanhados no grupo de diabetes do hospital universitário de Brasília. Brasília: Universidade de Brasília, 2016. Trabalho de Conclusão de Curso em Farmácia.

24. Melo DO. Avaliação das interações medicamentosas potenciais para pacientes internados na clínica médica do hospital universitário da USP visando à elaboração de instrumento para identificação de eventos adversos a medicamentos injetáveis. São Paulo: Universidade de São Paulo, 2010. Tese em Fármaco e Medicamentos. 